\title{
POWER OF POSTS: A QUANTITATIVE ANALYSIS OF FACEBOOK ELECTION CAMPAIGNING INTERACTIONS
}

\author{
MOHAMMAD ADIB KHAIRUDDIN
}

(Received 20 April 2017; first published online 5 July 2017)

\section{Mathematics subject classification: primary 00A06.}

Keywords and phrases: online election campaigning, Facebook page, control chart, interaction strength plot, stickiness level chart.

Media reports and past studies consider social media to be a valuable campaigning channel for political parties and candidates, especially during an election. Studies suggest that when used along with traditional campaigning methods such as advertisements on mass media and major rallies, social media increases the chances for candidates to win more support. Most research on social media election campaigning is more qualitative than quantitative. Furthermore, the focus of the small number of quantitative papers is on the use of Twitter. The limited number of papers that discuss Facebook use data coming from western democratic countries such as the United States and the United Kingdom. Moreover, these papers treat the bare count of likes recorded on the candidates' or the parties' Facebook as indicators of support and predictors for votes. This research [1] is about finding ways of measuring the effectiveness of using Facebook pages (FPs) for election campaigning, concentrating on the interactions that occur between the candidates and the public (users).

I use original data captured directly from the FP of selected candidates' campaigning in the 2013 Malaysian General Election (MGE13). The main reason for using Malaysia as a case study is the sudden change in the political landscape of the country. Unlike the earlier elections that have been dominated largely by the ruling coalition, the results of the MGE13 exhibit a sizeable shift to a more viable opposition in Malaysia. Media and scholars attribute this shift to the extensive use of internet and social media in the MGE13 campaign. The captured MGE13 data indicates that the success rate is indeed higher for candidates who are active (based on the bare count of posts published on his/her FP) and popular (based on the bare count of likes acquired on his/her FP). My research studies, in the main, the passive interactions (the users

Thesis submitted to RMIT University in September 2016; degree awarded on 5 January 2017; senior supervisor Asha Rao, associate supervisor John Postill.

(c) 2017 Australian Mathematical Publishing Association Inc. 0004-9727/2017 \$16.00 
liking a post published on a candidate's FP) with the aim of finding a more effective measure than bare count.

The research starts with statistically identifying and evaluating passive interactions. Results of the regression analysis imply that the response from users (in the form of likes) depends on the attractiveness of the posts, with some posts being more attractive than others. To study what makes these posts more attractive, I start with the use of cumulative sum charts, which have been used very effectively in the health industry. The cumulative sum chart, which has been termed (passive) interaction strength plot (P_IntS), presents a novel approach to measuring this attraction. P_IntS incorporates the variability of a candidate's posting, as well as the probability of the posts gaining the appropriate number of likes, in line with the possibility that a candidate's post could affect the number of likes received. The P_IntS chart reveals that almost $60 \%$ of the FP used in the MGE13 underperformed based on the steady negative progression of their P_IntS scores.

The P_IntS chart, however, does not accurately represent FPs that managed to grab a significant number of likes while actually publishing very few posts. Thus, other properties of the posts are explored that might affect the attraction or the stickiness of the posts. An investigation of the frequency and timing of the post uncovers correlations between events happening offline with the number of posts published as well as the number of likes gained. Steered by this, the analysis window has been narrowed down from 33 days to 16 days. For this interval, the posts that are sticky (posts that can incite the maximum number of likes) are isolated, and the sentiment of these posts are uncovered, so as to determine the possible reason for their stickiness. As a large number of posts are written using non-English (Malay) words, a new keyword list has been created that contains Malay sentiment words. Taking the importance of sentiment into account, the next graph, the stickiness level chart (SLC) measures the stickiness of the FP by using the likelihood of the sticky posts being posts containing positive or negative sentiment. The SLC highlights that the stickiness of the FP does depend on the sentiment of the sticky posts.

As a comparison, the interactions that occur in a similar number of FPs from the 2013 Australian Federal Election (AFE13) campaign have been captured and investigated. Based on the P_IntS chart, SLC graph and assessment of the sentiment of the posts in the AFE13 data, several similarities have been noticed as well as differences between the two data sets. One key similarity is that for both data, many FPs deemed active or popular (based on the bare count) underperformed, compared to the posts which were less sticky. On the other hand, in the MGE13 data there is more correlation between the offline events with online activity than in the AFE13 data. Also, unlike the MGE13 data, more posts with sentiment (positive or negative) are sticky in AFE13 data. The comparisons in general suggest that the interaction rate for FP campaigning is more conspicuous in the MGE13 than the AFE13.

The research was continued to analyse another type of interaction that exists on FPs. Different to the like feature, comment is another way for a user to actively interact with the candidate (post). Even though it has been noticed that half of the comments 
received by the sticky posts published on popular FPs are positive, there are still many aspects of comments that need to be examined further. This is work in progress and a number of questions are presented that are suitable for future work.

In conclusion, this study shows that including the properties of the posts in measuring FP interactions results in more reliable statistics than the bare counts. Besides being a tool for assessing interactions, P.IntS and SLC have the potential of strategically guiding the use of social media in an election campaign.

\title{
Acknowledgements
}

I would like to express my utmost gratitude to my esteemed supervisor, Professor Asha Rao, and a sincere thank you to Dr John Postill.

\section{Reference}

[1] M. Khairuddin, 'Power of posts: a quantitative analysis of Facebook election campaigning interactions', Doctor of Philosophy, Mathematical and Geospatial Sciences, RMIT University, 2016.

\author{
MOHAMMAD ADIB KHAIRUDDIN, \\ Jabatan Sains Komputer, FSTP, \\ Universiti Pertahanan Nasional Malaysia, \\ Kuala Lumpur, Malaysia \\ and \\ Mathematical and Geospatial Sciences, \\ School of Science, RMIT University, \\ Melbourne, VIC, Australia \\ e-mail: adib@upnm.edu.my
}

